\title{
La zona di transizione fra mantello e nucleo terrestri è stratificata: sua probabile origine
}

\author{
P. CALOI
}

Ricevuto il 10 Dicembre 1966

Riassunto. - Si portano nuove testimonianze comprovanti l'esistenza di una zona di transizione (comportantesi come solida) fra quella cloo era ritenuta la base del mantello, a $2900 \mathrm{~km}$ di profonditi (superficio di Wiechert-Gutenberg), e il nucleo terrestre. Nel 1964 l'autore provò che tale zona ha lo spessore approssimativo di $160 \mathrm{~km}$, e che in essa la velocita di propagazione delle onde longitudinali decresce gradualmente. In ulteriori determinazioni, che vengono qui presentate, si valuta che, a contatto con il nucleo la velocita delle onde $P$ sia dell'ordine di $10 \mathrm{~km} / \mathrm{sec}$. Analoga flessione consegue per le onde trasversali.

Appare chiara la stratificazione della zona di transizione, come lo dimo. stra la registrazione di impulsi sucessivi, nettamente intervallati, provenienti da discontinuità comprese fra la baso del mantello (superficio " $c "$ ) e l'inizio del nucleo (superficio " $N$ )).

Per la giustificazione di tale zona, si ritiene idonea la teoria di Ramsey. Se il mantello inferiore - conformemente alle conclusioni dei geochimici consiste di un sistema a più componenti (p. es. ossidi compatti di magnesio, silicio e ferro, come propone Birch), la zona, nel suo complesso, corrisponderebbe alla transizione del mezzo, per pressione, dalla fase molecolare alla fase metallica, le discontinuità corrispondendo al completo transito di fase dei diversi componenti. Alla transizione totale dell'ultimo componente, conseguirebbe la formazione del gas degenere di Fermi. Per questa via verrebbe spiegata la caduta di velocità delle ondo elastiche, legata al forte aumento della densità e alla progressiva diminuzione dolla rigidita.

Come l'autore ha gia provato nel 1964 , l'esistenza della zona di transizione risolve - fra l'altro - le difficoltà inerenti alla propagazione delle onde longitudinali nella zona d'ombra.

Naturalmente, il chiarimento della costituzione del mantello inferiore, porta con se ovvie revisioni anche per la parte più interna della Terra, comprendente il nucleo esterno e il nucleo interno. 
SUmMary. - New evidences about the existence of a transition zone between the lower mantle and the core are carried.

In 1964, the author proved this zone is about $160 \mathrm{kms}$ thick and velocity of elastic waves decrease in it gradually. Now he judges the velocity of $P$ wave, at the bottom of the transition zone, of $10 \mathrm{~km} / \mathrm{sec}$ order.

The zone results layered, how the record of intervalled, successive pulses proves.

For the giustification of this zone the Ramsey's theory is trought fit

1. - In una Nota presentata fra gli Atti dell'Accademia dei Lincei nel Giugno del $1964\left(^{(1)}\right.$ avevo provato che le anomalie di propagazione delle onde longitudinali, legate alla così detta zona d'ombra, possono ottenere chiara spiegazione se si ammette che il mantello terrestre non confina bruscamente con il nucleo esterno, bensì ne è separato da una zona di transizione, caratterizzata da velocita decrescenti delle onde longitudinali (e trasversali). Sulla base dello studio delle onde $P c P$, $S c P, P c S$, ScS, registrate da strumenti a breve periodo, concludevo che la zona di transizione su detta doveva presentare uno spessore di $160 \mathrm{~km}$. ca. Le onde riflesse dalla base della zona stessa, indicavo con il simbolo intermedio " $N "\left(P_{N} P \ldots S_{N} S\right)$ a significare che essa costituiva l'effettivo inizio del "Nucleo".

La validità di quelle conclusioni è dimostrata dalle nuove testimonianze che qui presento (vedi Figgr. 1, 2, 3, 4).

Oltre a confermare quanto è stato sopra riassunto, dal loro esaune possono essere tratte ulteriori precisazioni.

1) Non solo esiste la zona di transizione tra mantello inferiore e nucleo, ma essa è quasi certamente stratificata. Gli impulsi che seguono le $S c S$, p. es., $\left(S_{N} S, \ldots S_{N} S\right)$ possono spiegarsi solo come echi su superficie rillettenti, poste a quote diverse fra la superficie di Wiechert (" $c \|$ ) e il nucleo (" $N ")$.

2) Raramente gli apparecchi, di periodo proprio superiore ad 8-10 sec, registrano onde di tipo $S_{N} S$. Esse, assieme alle $S c S, \ldots$ appaiono chiaramente solo sulle registrazioni di sismografi a breve periodo.

Gli apparecehi a lungo periodo registrano onde $S c S$ con periodi di $10-15$ sec; e in esse si esaurisce il fenomeno della riflessione, quasi che il loro periodo fosse imposto dall'intera zona di transizione, che risponde 
all'onda incidente come la crosta terrestre risponde all'energia incidente per la formazione delle onde che io chiamo di Somigliana $\left(C_{1,1}\right.$, per es. $)\left({ }^{2}\right)$.

Soltanto l'energia associata ai brevi periodi, dopo parziale riflessione alla base del mantello (discontinuità di Wiechert), riesce a penetrare nella zona di transizione e a subire successive riflessioni nelle stratificazioni interne, l'ultima delle quali sara in corrispondenza della superficie " $N$ ".

3) Le $S c S$ presentano trascurabile componente verticale (Figg. 1, 3), mentre tale componente si fa sensibile per onde riflesse nell'interno e alla base della zona di transizione. Sembra quasi che le onde ScS siano di tipo $S H$, mentre penetrano e vengono riflesse nella zona di transizione onde di tipo $S T$.

Questo fatto spiega perchè le onde $S c P$ siano di piccolissima ampiezza (solo la componente $S V$ può trasformarsi infatti in onde $P$ ), mentre l'ampiezza della $P c S$ è sempre più marcata perchè le $P$, cambiando carattere nella riflessione, possono mutarsi soltanto in $\mathrm{SV}$ (Figg. 1, 3).

Dalla differenza $S c P$ - $P c S$ è possibile trarre il valore approssimativo della profondità ipocentrale.

Si potrà osservare che la differenza $S_{N} S-S c S$, pur diminuendo con la distanza epicentrale, presenta una diminuzione piuttosto lenta (si confrontino le registrazioni ottenute a Tolmezzo per distanze epicentrali dell'ordine di $1500 \mathrm{~km}$ con quelle ottenute, p. es., a Quetta per distanze epicentrali dell'ordine di $5700 \mathrm{~km}$ ).

La spiegazione è la seguente. In un mezzo stratificato, caratterizzato da velocità delle onde elastiche via via decrescenti, il tragitto di onde in esso rifratte sotto angoli d'incidenza via via crescenti, non differisce di molto da quello di onde incidenti pressochè normalmente, in quanto si verifica, nella propagazione verso il basso, un progressivo avvicinamento alla normale nel punto d'incidenza. A questo riguardo, dev'essere sensibilissima la diminuzione della velocità delle onde trasversali verso la base della zona di transizione, come è provato dal lungo persistere di onde di tipo $S_{N} S$, dopo la registrazione dell'ultimo impulso (vedi Fig. $1 \mathrm{~g}$, Tolmezzo).

Che la velocità delle onde longitudinali (e trasversali) diminuisca sensibilmente nella zona di transito, ho già provato in un precedente lavoro $\left({ }^{3}\right)$. 


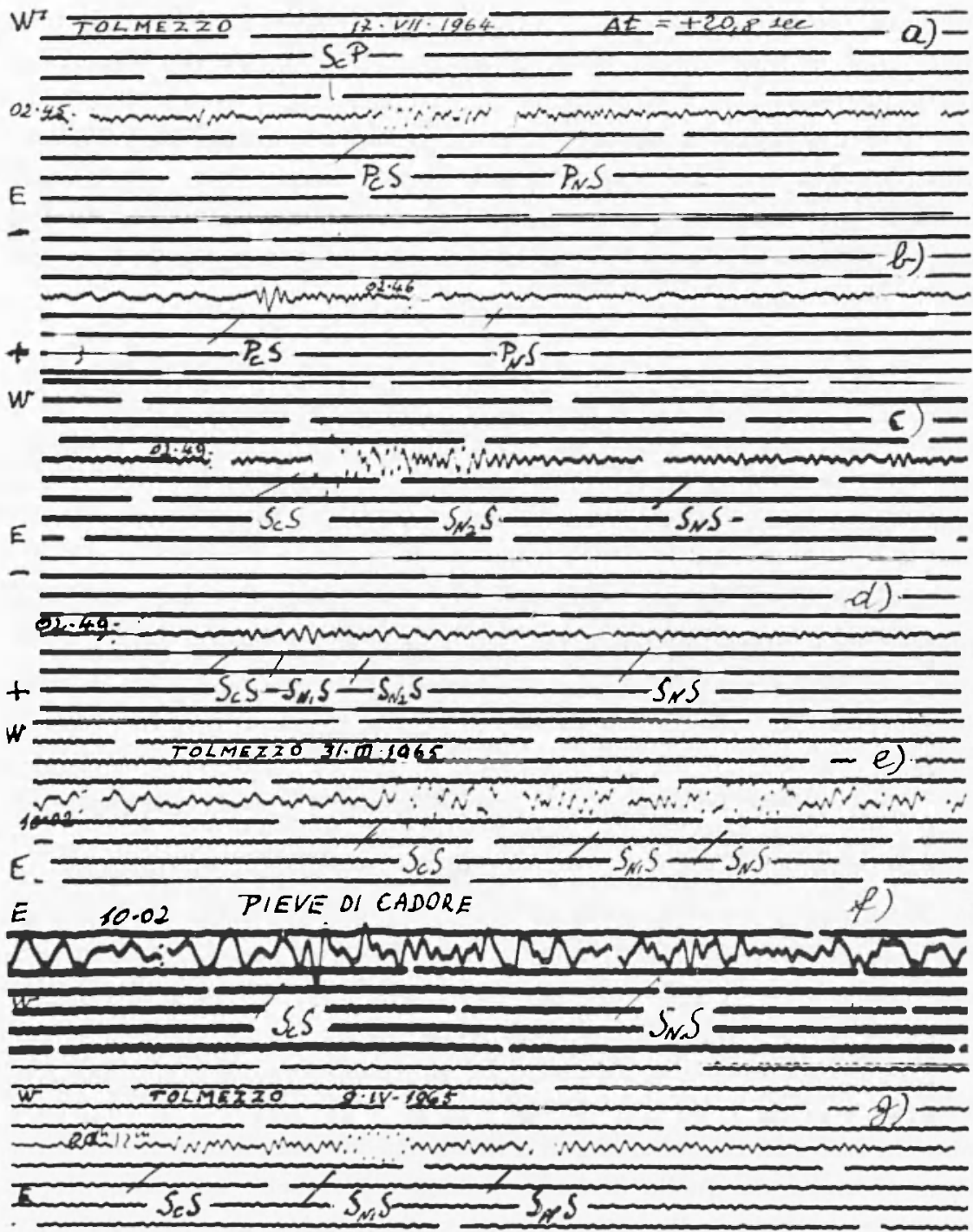

Fig. $1-17$.VII.1964 (Grecia $\div 38^{\circ}, 2 N ; 23^{\circ}, 7 \quad E ; \quad I I=02.34 .26,9$; $h=150 \mathrm{~km}$. a) b) c) d) registrazioni di Tolmezzo $(\Delta=1300 \mathrm{~km})$ : per le onde miste, sulla comp. vert. appare netta la sola $P c S$ (vedi b). La ScŚ, molto ampia sulla comp. oriz,, sembra avere predominanti i caratteri di S'I : nanca infatti il suo inizio sulla comp. vert.: negli impulsi suceessivi $\left(S_{N 1}, S, \ldots\right)$, appaiono chiari invece i caratteri della $S V$ (vedi $d$ ).

31.111.1965 (Grecia: $38^{\circ}, 4 N ; 22^{\circ}, 2 E ; \quad N=63 / 4$ Pas.; $I I=$ $09.47 .29 ; h=100 \mathrm{~km}(\mathrm{c}): e)$ registrazione di Tolmezzo, $\Delta=1165 \mathrm{~km}$; f) registrazione di Pieve di Cadore, $\Delta=1.200 \mathrm{~km}$.

9.IV.1965 (Grecia: $35^{\circ}, 1 N ; 24^{\circ}, 3 k ; M=5 ; H=23.57 .03,2$; $h=51 \mathrm{~km}): g)$ regristrazione di Tolmezzo, $\Delta \ldots 1570 \mathrm{~km}$. 
LA ZONA DI TRANSIZIONE FRA MANTELLO E NUCLEO TERRESTRI. HCC. 125

Si è visto che, il valore reale della velocità re alla base del mantello puó ottenersi dalla formula:

$$
v_{c}=\frac{r_{2}}{r_{0}} V
$$

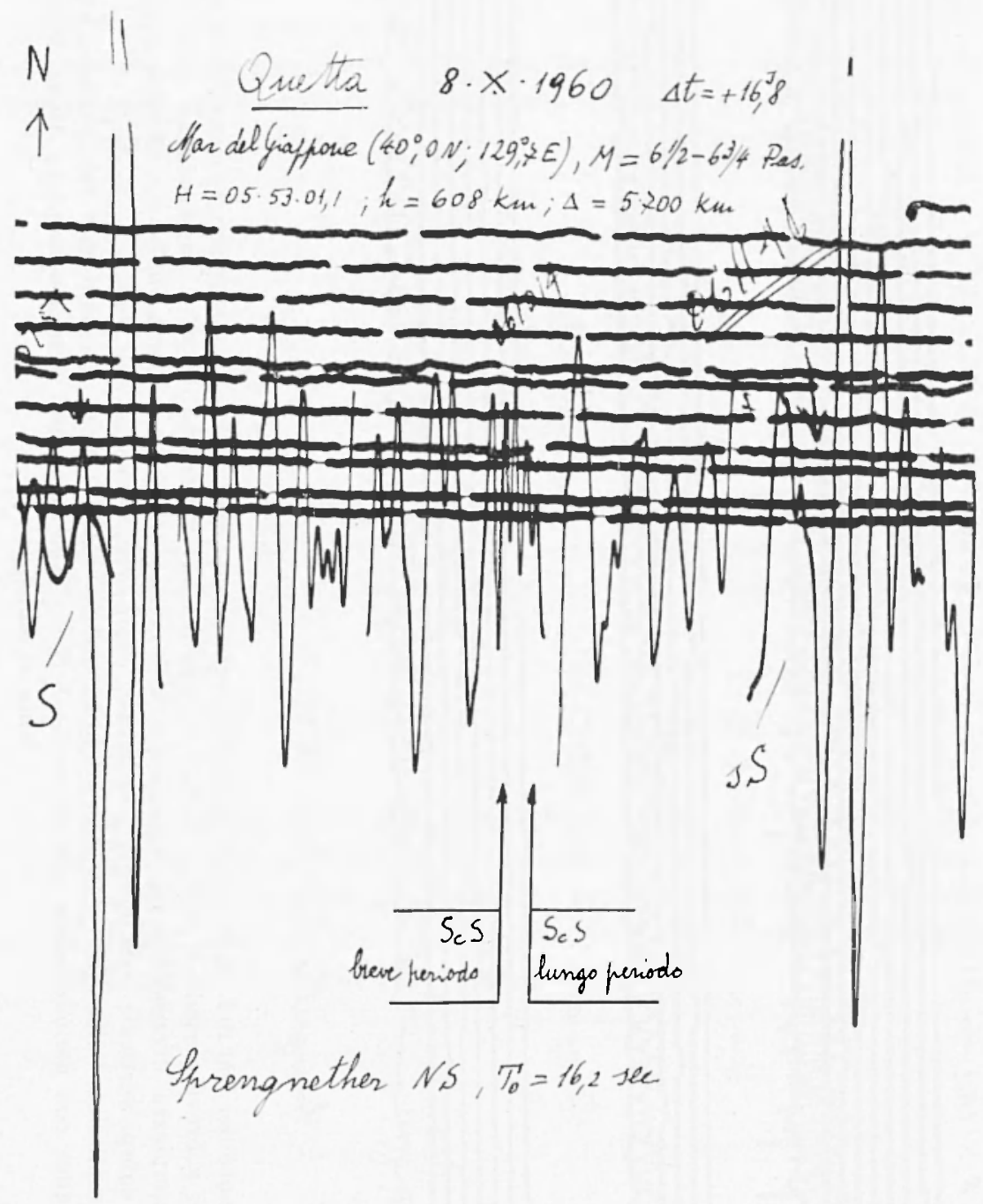

Fig. 2 - Terremoto Mar del Giappone $\left(40,00 N ; 129,{ }^{\circ} 7\right.$ E) $)$ Registraziono di Quetta (NS Spregnether lungo periodo). Ise $S, s S$ a lungo periodo ap. paiono molto sviluppate, nei confronti delle ScS a lungo periodo, sulla comp. YS con periodo proprio di $10 \% 2$. Le componenti Spregnether a breve periodo $\left(T_{0}=18,8\right)$ della stessa stazione, registrano invece, in maniera predominante, sopratutto le $S c S, S_{N} S$ con periodo di $3^{*}$ ca (vedi Fig. 3). 


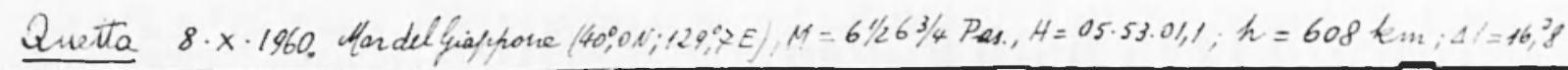

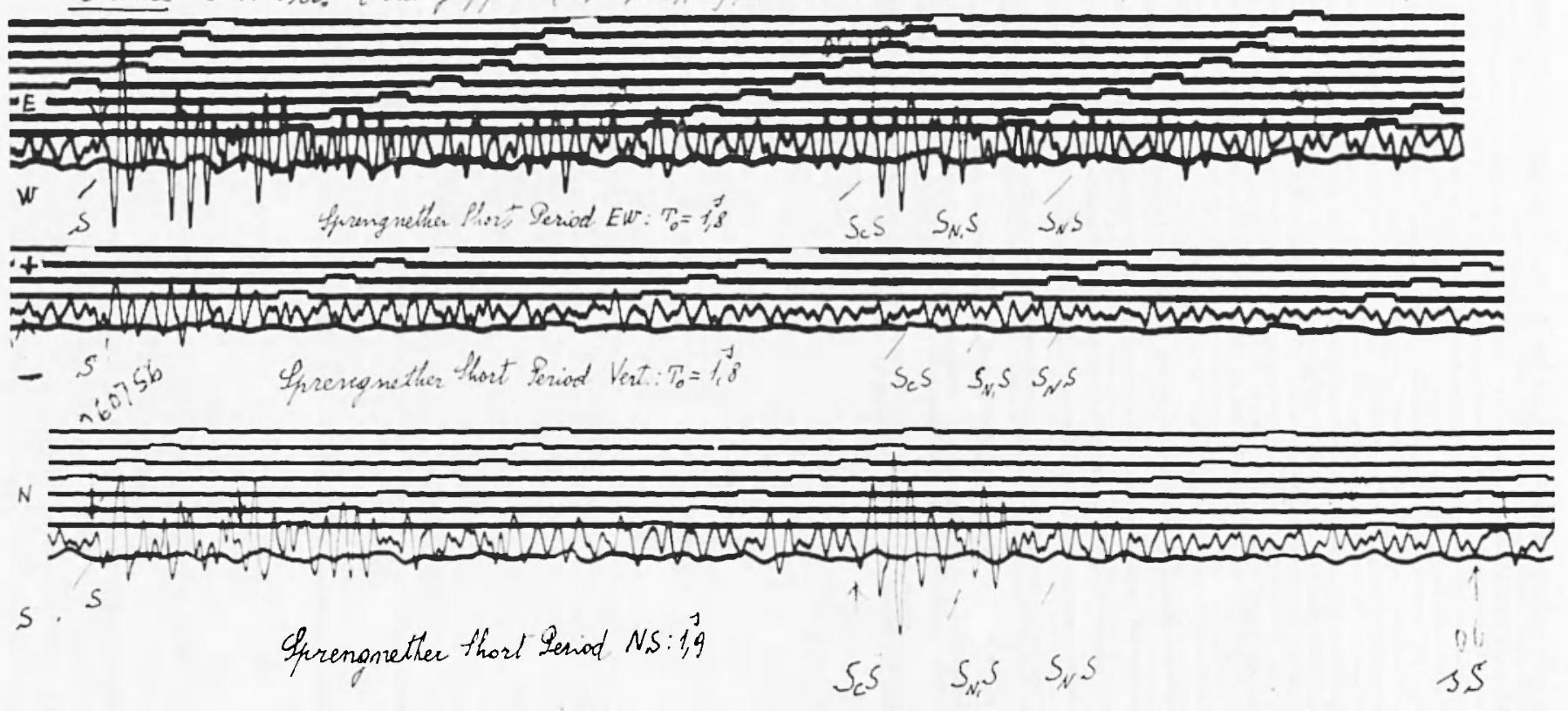

Fig. 3 - Terremoto del Mar del Giappone (8. X. 1960), registrazioni di Quetta, su componenti Spregnether a l,reve periodo. Sugli apparecehi a breve periodo le $S c S, \ldots S_{N} S$ - con periodi dellordine di $3^{s}$ - predominano sulle $S$ (vedi $X S$ ). Gli impulsi, di ampiezza decrescente, si susseguono a brevi intervalli, a riprova della stratifienzione della zona di transizione. La comp. verticale is di piccola ampiezza e, comunque, associata alle $S_{N} S$.

Le $S$ e le $S S$, amplissime sulla $N S-1\left(8,2\right.$ (v. Fig. 2), con periodo di $12^{8}$ ca, mancano, con tale periodo, sulla $N S^{--18,8 .}$ Solo la $S$ vi figura con periodo molto più breve. l.e $S c S, \ldots S_{N} S$ sembrano quindi la risposta libera degli strati della zona di transizione. 
dove $r_{c}$ è il raggio medio dello strato-base del mantello, $r_{0}$ il raggio terrestre e $V$ la velocità apparente, tratta dalla traiettoria media dei tempi di tragitto delle onde $P$ nella zona d'ombra.

Nel lavoro citato $\left({ }^{3}\right)$, il valore di $V$, conseguente all'applicazione del metodo dei minimi quadrati ai 99 tempi di tragit to di onde $P$ registrate nella zona d'ombra, relativi a 27 terremoti a profondità normale, tratti dall' Inter'national Seismological Summary " (24 del 1951 e 3 del 1952), era risultato:

$$
Y=25 \mathrm{~km} / \mathrm{sec} c \mathrm{a}
$$

Fatto $r_{c}=3300 \mathrm{~km}, r_{o}=6370 \mathrm{~km}$, la [1] dà:

$$
v_{c}=12,95 \mathrm{~km} / \mathrm{sec} \text { ca. }
$$

Un'ulteriore applicazione a 113 valori per le $P$, registrate nella zona d'ombra, tratti dall'I.S.S., e relativi a terremoti a profondita normale, 5 del 1955 e 8 del 1956 , ha condotto ai seguenti valori, per $V$ e $v_{c}$ :

$$
V=24,7 \mathrm{~km} / \mathrm{sec}, \quad v_{c}=12,85 \mathrm{~km} / \mathrm{sec} .
$$

Ho effettuato nuove analoghe determinazioni di $V$ e $v_{c}$, per le $P$ relative alla zona d'ombra di sei terremoti singoli.

In queste determinazioni però mi sono limitato alle registrazioni delle onde $P$ ottenute nella prima parte della zona d'ombra, allo scopo di usufruire di dati d'osservazione più attendibili.

La media dei sei risultati, separatamente ottenuti, fatto $r_{c}=$ $3300 \mathrm{~km}$, è stata la seguente:

$$
Y=21,73 \mathrm{~km} / \mathrm{sec}, \quad v_{c}=11,49 \mathrm{~km} / \mathrm{sec} \pm 0,49
$$

mentre l'elaborazione complessiva delle cennate $P$, relative agli stessi terremoti (Fig. 5), ha condotto a:

$$
V=21,43 \mathrm{~km} / \mathrm{sec}, \quad v_{c}=11,3 \pm \mathrm{km} / \mathrm{sec} \pm 0,29
$$


Terremoto di Formosa del 12-III-1966.

$\left(24^{\circ}, 1 N-122^{\circ}, E ; \quad H=163121.8 ; h=63 \mathrm{~km} ; \quad \lambda=7^{1} \cdot 2-7^{3 / 1}\right)$.

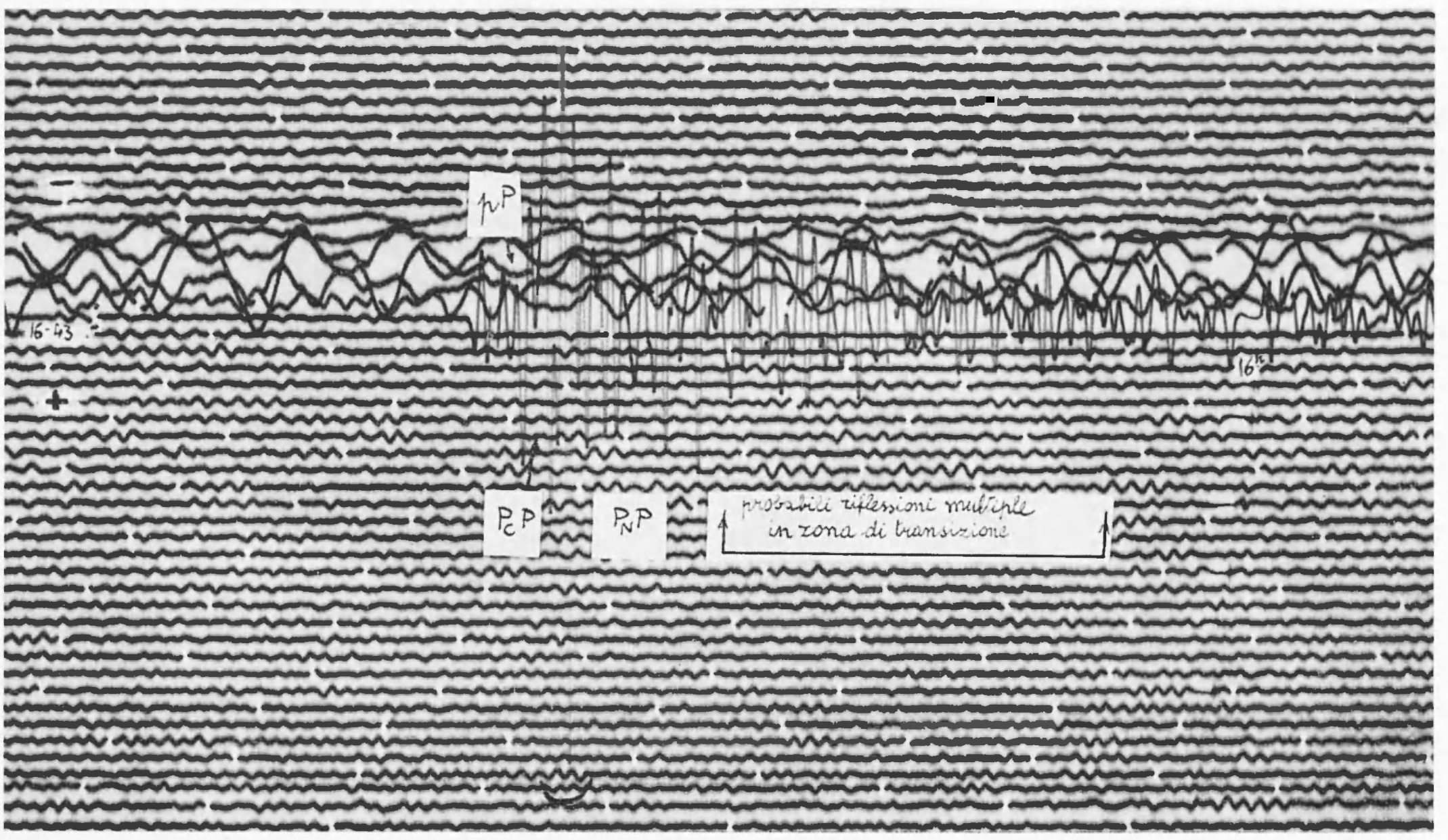

Fig. 4 - Vert. Vajont 12-III-1966 ( $\left.\Delta t=+5^{s}, 1 ; A=9600 \mathrm{~km}\right)$. 


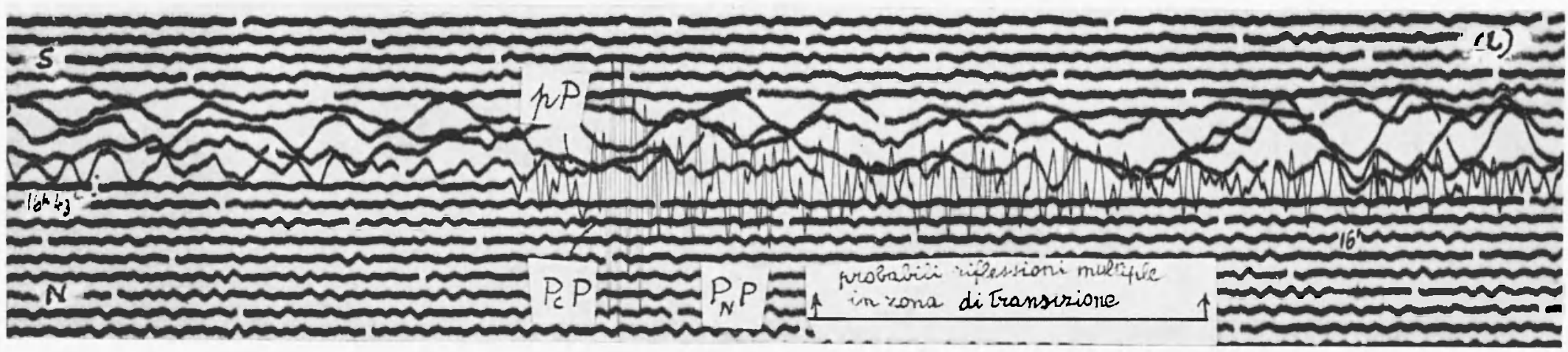

Fig. $4 a-$ Vajont comp. NS.

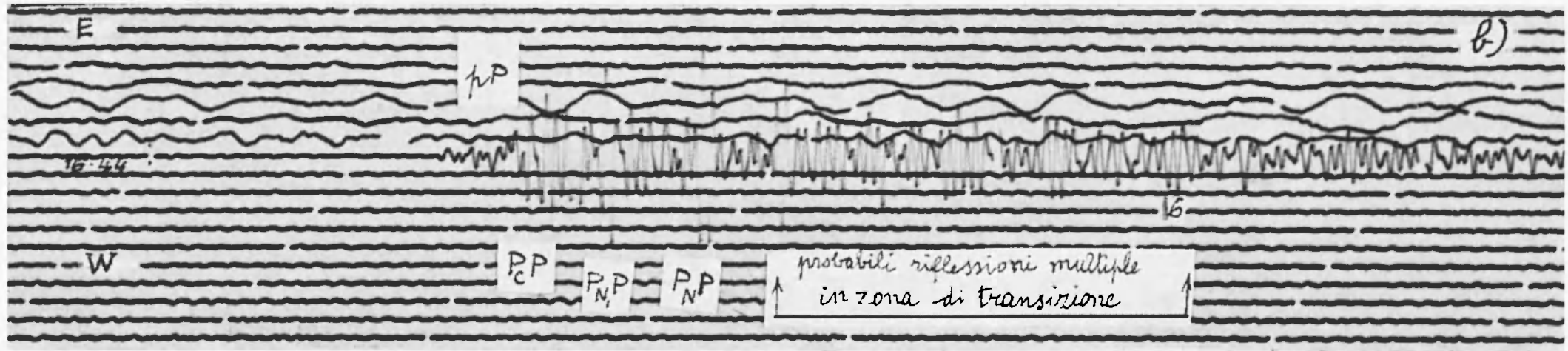

Fig. $4 b$ - Pieve di Cadore; comp. $E W\left(\Delta t=-46^{8}, 4 ; A=9600 \mathrm{~km}\right)$.

Gli impulsi seguenti le $P_{N} P$ per la durata di circa un minuto - rimasti finora senza plausibile interpretazione, specie per le distanze fra 9000 e $10000 \mathrm{~km}$ - possono essere spiegati come ritorni in superficie di onde longitudinali che, dopo incidenza contro la superficie di IViechert, sotto angoli prossimi a $90^{\circ}$, vengono catturati dalla zona di transizione e costretti a propagarsi nelle sue stratificazioni, sotto forma di riflessioni multiple. 


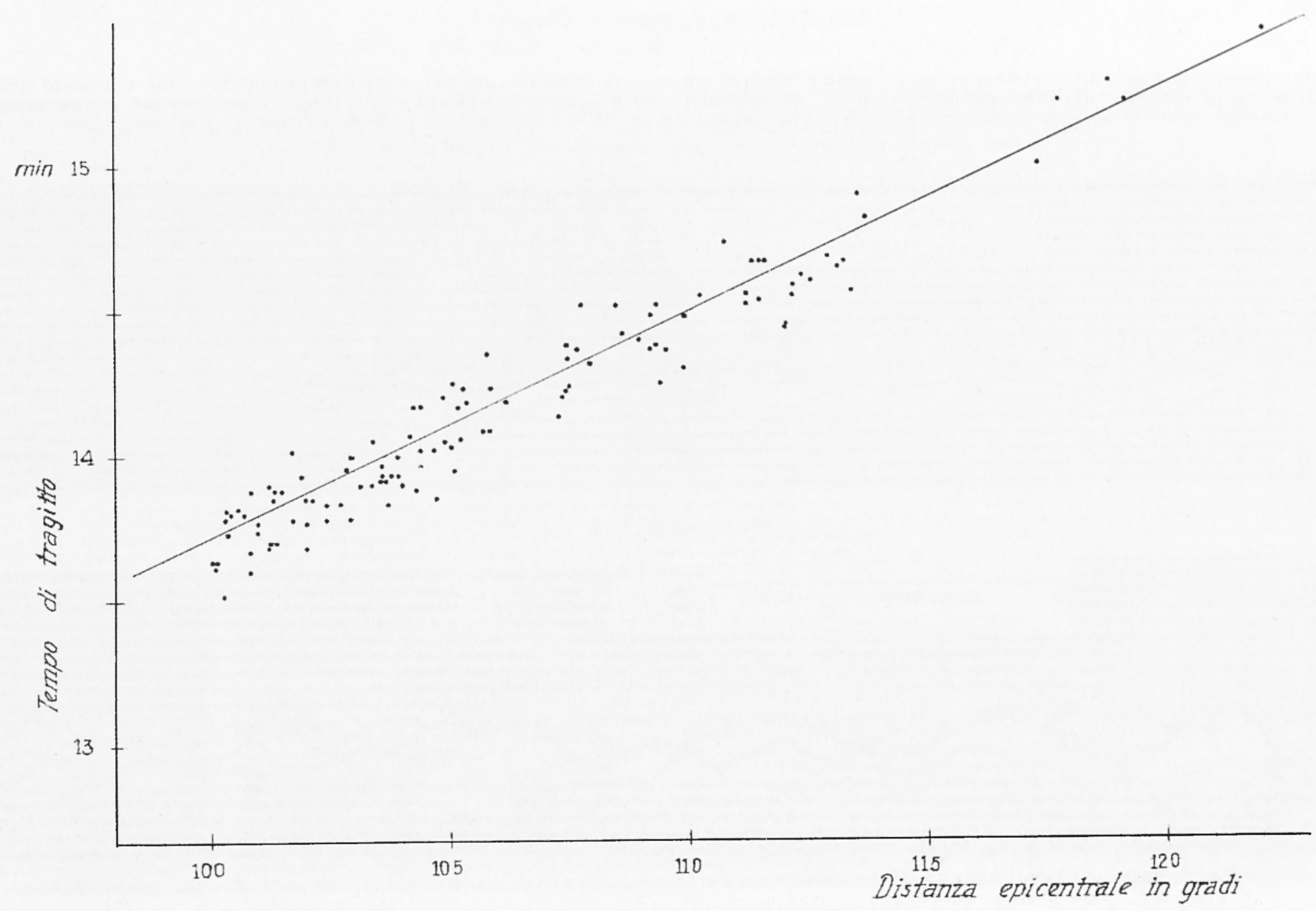

Fig. 5 
Il valore cosi ottenuto per la $v_{c}$ è quindi alquanto inferiore al vallore di $13,75 \mathrm{~km} / \mathrm{sec}$, velocità che raggiunge alla base del mantello, in corrispondenza della discontinuità " $c$ ". Se poi si considera che la $v_{c}$ cosi ottenuta rappresenta forzatamente il valore medio-massimo della velocità delle onde longitudinali in quella parte della zona di transizione immediatamente al di sotto della discontinuità di Wiechert " $c$ ", si può ritenere che tale velocità presenti valori ancora minori, e tenda a decrescere gradualmente verso la superficie " $N$ " (in prossimità della quale la velocità delle onde longitudinali è probabilmente dell'ordine di $10 \mathrm{~km} / \mathrm{sec}$ ).

2. - Prima di passare al problema concernente la formazione della zona di transito fra mantello e nucleo, è bene esaminare, alla luee delle nuove acquisizioni, i risultati di precedenti ricerehe sulla natura del nucleo esterno, nell'ipotesi di una separazione brusca della base del mantello dal nucleo.

Particolarmente notevoli, a questo riguardo, i lavori di H. Honda a collaboratori.

a) Nel 1934, H. Honda ( $\left.{ }^{4}\right)$ aveva esposto una sua teoria sulla rigidità del nucleo, dedotta dall'esame delle $S c S$. Nel 1940, in collaborazione con $\mathrm{Y}$. Hasaya $\left({ }^{5}\right)$, egli applica la teoria esposta allo studio delle $S c S$, associate al terremoto profondo del Mar del Giappone del 13 Novemble 1932 ( $\left.\varphi=43^{\circ} 5 \tau^{\prime} \mathrm{N} ; \lambda=137^{\circ} 25^{\prime} \mathrm{E}, h=320 \mathrm{~km}\right)$.

I calcoli sono stati condotti sulle osservazioni ottenute in 12 stazioni sismiche giapponesi, con distanze epicentrali variabili fra 336 - $1380 \mathrm{~km}$. Il rapporto fra le $S c S$ osservate e le $S c S$ calcolate (SeS oss./SeS cal) ha portato alla conclusione che il valore delle SeS osservate, è, in media, la metà di quello delle $S c S$ calcolate, quando il nucleo viene considerato liquido, mentre, nel caso di un nucleo solido, la $S c S$ osservata risulta, in media, 9 volte maggiore della SeŚ ralcolata.

I risultati dell'osservazione si approssimano quindi a quelli alcolati molto di piǹ quando il nucleo è supposto liquido, che non quando è considerato solido. Inoltre, mentre riesce difficile spiegare come l'ampiezza osservata possa risultare molto maggiore di quella calcolata, al contrario viene spontaneo attribuire la minore ampiezza degli spostamenti osservati, nei confronti di quelli calcolati, allassorbimento che l'energia delle onde sismiche subisce durante il lungo pereorso delle ScS.

Gli Autori ne concludono che la rigidità della parte esterna del nucleo è pressoché nulla. 
Conclusione logica, naturalmente, data l'enorme differenza che le osservazioni presentano nei confronti dell'ipotesi di un nucleo solido. Però, come vedremo, è esagerato attribuire al percorso nel mantello l'assorbimento di oltre il $50 \%$ dell'energia associata alle SeS'.

b) Successivamente, Honda, con la collaborazione di Ito $\left({ }^{6}\right)$, riprese la questione. Essi studiano la natuma del nucleo esterno, sulla base delle osservazioni delle ampiezze delle onde $P$, S, $p P$, ScS di terremoti profondi. Si preoccupano, in questa ricerca, di tener conto altresi del meccanismo del terremoto in esame [21.IV.1939; $H=04^{\mathrm{h}} .29^{\mathrm{m}}$ ca; $\varphi=47^{\circ} 36^{\prime} \mathrm{N}, \lambda-140^{\circ} 12^{\prime} \mathrm{E} ; h=530 \mathrm{~km}$ all'ipocent $h$, che condiziona la propagazione dell'energia sismica nelle varie direzioni. A noi interessano qui le conclusioni tratte dagli Autori sulla propagazione delle onde $S c S$. Essi trovano che, supponendo il nucleo esterno liquido, i calcoli portano ad un'ampiezza iniziale delle $S c S$ pari a circa il $60 \%$ di quella delle onde $S$, non di molto superiore ai dati forniti dall'osservazione; mentre nell'ipotesi di un nucleo esterno solido, l'ampiezza delle ScS risulterebbe teoricamente soltanto circa il $5 \%$ di quella delle onde $S$.

Conclusione ovvia - e naturalmente giusta - anche per questa via, la natura elastica del nucleo esterno appare quella di un liquido.

Peró, anche questa volta, la pur lieve discrepanza con le osservazioni non può essere griustificata con un transito repentino fra mantello e nucleo.

c) Un'ulteriore ricerca fu condotta sotto la direzione di Hondir. Le precedenti indagini di Honda e Hasaya, e Honda e Ito sulle onde ScS erano state fondate sulle leggi della riflessione dell'onda $S$, supposta armonica piana. Poiché, per terremoti profondi, l'onda ScS registrata nelle stazioni vicine all'epicentro apparve ad Honda con gli aspetti di un'onda solitaria, consistente di una singola oscillazione completa, ricerche più recenti di Honda, Sima e Nakamura (7) furono rivolte ad ottenere l'espressione del movimento per onda $S V$ riflessa alla superficie di separazione fra solido e liquido (o solido e solido), allorquando un'onda impulsiva $S V$, emessa da una sorgente all'interno del solido, incide sulla superficie di separazione.

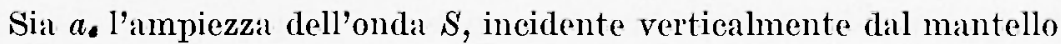
sulla superficie del nucleo e $a_{r}$ l'ampiezza dell'onda riflessa. Riserviamo l'indice 1 al nucleo e l'indice 2 al mantello; se $\varrho$ e $v_{s}$ rappresentano la 
densitì e la velocità delle onde trasversali rispettivamente, poniamo:

$$
\begin{aligned}
& \underline{o}_{1}=9,7 \mathrm{gr} / \mathrm{cm}^{3} \quad,\left\{\begin{array}{l}
v_{1,8}=0 \text { (nucleo liquido) } \\
v_{1,8}=4,6 \mathrm{~km} / \mathrm{sec} \text { (nucleo solido) }
\end{array}\right. \\
& \varrho_{2}-6,66 \mathrm{gr} / \mathrm{cm}^{3} \quad, \quad v_{2, s}=7,25 \mathrm{~km} / \mathrm{sec} \text {. }
\end{aligned}
$$

Per un'onda piana $S$, incidente normalmente sulla superficie limite del nucleo, supposta piana, si ha:

$$
\frac{a_{r}}{a_{e}}=\frac{\varrho_{1} \cdot v_{1, s}-\varrho_{2} \cdot v_{2, s}}{\varrho_{1} \cdot v_{1,8}+\varrho_{2} \cdot v_{2, s}} .
$$

I valori di $a_{r_{i}} a_{e}$ sono illustrati nella Fig. 6, calcolata da llonda e collaboratori per $\varrho_{1} / \varrho_{2}$ compreso fra 1,0 e 2,2 e $v_{1, s} / v_{2, s}$ variabile fra 0 e 1,0 .

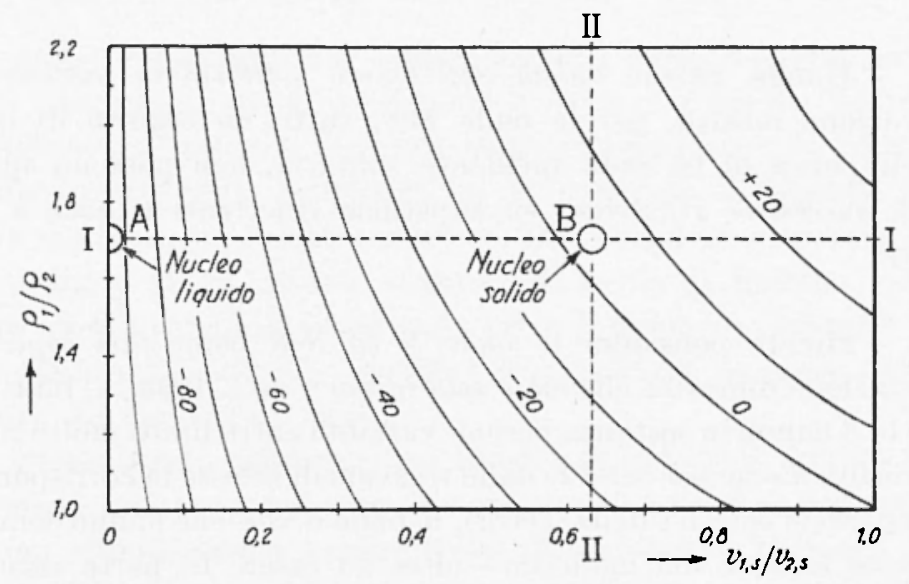

Fig. 6 - Valori di $a_{r} / a_{e}(\%)$, secondo Honda, Sima e Nakamura.

In Figura 6 la linea I-I rappresenta $\varrho_{1} \varrho_{0}=1,71$, valore corrispondente a quelli generalmente accettati di $\varrho_{1}=9,7$ e $\varrho_{2}=5,66 \mathrm{gr} / \mathrm{cm}^{3}$. La linea II-II rappresenta $v_{1,8} / v_{2, s}=0,63$ valore che - posto uguale a $1 / 4$ il rapporto di Poisson - corrisponde a $v_{1, s}-4,6 \mathrm{~km} / \mathrm{sec}$ (fat to $v_{1, p}=8,0 \mathrm{~km} / \mathrm{sec}$ ), nell'ipotesi di un nucleo solido.

Il punto $B$ (intersezione di I-I con II-II) griace nel dominio della figura in cui la fase viene rovesciata e $a_{r} / a_{e}$ è molto piceolo (prossimo allo zero). Poiché i risultati delle precedenti indagini sulle onde ScS dei terremoti profondi dimostrano chiaramente che $a_{r} / a_{e}$ differisce poco da 1, uli Autori ne conchudono che l'ipotesi di un nucleo solido è da escludere. 
Il punto A corrisponde alle condizioni $\varrho_{1} / \varrho_{2}=1,71, v_{1,8}=00$ $a_{r} a_{e}=1$, ciò̀ al caso di un nucleo esterno liquido; ed è questo il caso accettato dagli Autori.

Honda e collaboratori considerano un terzo caso; quello in cui $a_{r} / a_{e}=-0,6$ oppure $-0,8$ e $\varrho_{1} / \varrho_{2}=1,71$. A queste condizioni, la rigidità della parte del nucleo interessata dovrebbe essere 0,06 oppure 0,0.1 volte quella del solido corrispondente a $B$. Gli Autori, poiché questi valori dellia rigidità non si possono conciliare con quelli di un qualunque corpo solido normale, negano qualsiasi attendibilità al caso in questione. A mio avviso, tenuto conto della probabile natura della zona di transizione (avente le caratteristiche del gas anomalo di Fermi), è invece questo il easo più interessante esaminato dai tre giapponesi.

A proposito dei risultati delle indagini di Honda e collaboratori, vanno fatte le seguenti precisazioni.

10. - Honda ritiene l'onda ScS essere solitaria in partenza. Ora l'osservazione mostra, per le onde $S c S$, tutto un seguito di impulsi che, nell'ipotesi di an'onda incidente solitaria, non possono spiegarsi che con successive riflessioni su superficie riflettenti situate a quote inferiori.

20. - Ionda considera le onde $S$ ed $S c S$ come fasi coperiodali. I'osservazione dimostra che ciò è solo in parte vero. Risulta, infatti, che mentre le $S$ hamo in sostanza periodi variabili entro limiti molto ristretti (come risulta anche per tutte le onde trasversali riflesse in corrispondenza della superficie esterna della Terra), le onde $S c S$ - che hanno commque interessato la base del mantello-oltre ad essere in parte associate a periodi dell'ordine di quelli delle $S$ (dalle quali derivano, vedi Figr. 2), mostrano tutta una gamma di periodi con netta prevalenza di quelli brevi, questi ultimi potendo essere rivelati solo da sismografi a breve periodo (vedi Fig. 3). Fra i tanti esempi, basterà citare quello ottenuto a Quetta in occasione dellar registrazione del terremoto del Mar del Giappone dell'8 Ottobre 1960 (Figg. 2 e 3).

In detta stazione funzionano due componenti $N S$ Sprengnether, una a lungo periodo $(16,2 \mathrm{sec})$, l'altra a breve periodo $(1,9 \mathrm{sec})$. Mentre la prima dì amplissime onde $S$ (con periodo dell'ordine di 12-14 sec) e onde dello stesso tipo riflesse dalla superficie esterna della Terra (sS), con registrazione di $S c S$ di relativa piccola ampiezza, la componente a breve periodo registra in modo netto esclusivamente le onde $S c S$ (con periodi dell'ordine di 2,5-3 sec). 
Da ciò consegrnono due conclusioni:

I. - Non è consentito un confronto di ampiezze fra le s s scs. tale confronto risultando foratamente parziale.

II. - Il prevalere di oscillazioni a breve periodo nelle ScS sta a significare che la loro riflessione avviene in un mezzo stratificato in cui la rigidità è fortemente scematr. Il fatto poi che, specie nelle regristrazioni a piccola distanza epicentrale, le onde ScS siano seguite da tutta una serie di impulsi a breve periodo sta a provare che il mentello non termina a $2.900 \mathrm{~lm}$ ca (come generalmente si ritiene), ma che esso ì continuato da un mezzo a rigidità ridotta, probabilmente stratificato, di nno spessore complessivo dell'ordine di 160-170 $\mathrm{km}$.

In complesso, sulla ricerche di Honda e collaboratori, possiamo fare le seguenti osservazioni:

a) Le conchusioni eui giunge Honda, nelle ricerche antiche e recenti, conclusioni che lo conducono a ritenere il nucleo liquido, non contraddicono all'esistenza di questo strato di transizione. In effetti, esse sono state tratte nella constatazione dellesistenza di reazioni paragonabili a quelle di un liquido, il che risulta conforme a quanto è stato detto al punto II. Se, infatti, si invoca la teoria di Ramsey, possiamo ritenere questo strato di transizione come il graduale passaggio da una fase molecolare ad una fase metallica per pressione, la quale finisee col confinate con lo strato liquido.

A questo strato puó benissimo competere la piccola rigidità trovata da Honda e coll. (v. e) nel caso di $a_{r} / a_{e}=-0.6(-0.8)$, e da essi ritenuta non rispondente a nessun solido in condizioni normali. Infatti, nella transizione, per pressione, del basso mantello verso il gas degenere di Fermi (v. n. 3), la rigidità subisce una progressiva, fortissima diminuzione.

ק) Già in precedenza, specialmente dalla sig.na Lehmann, era stato notato che le onde longitudinali registrate nella zona d'ombra damno tempi di tragitto sensibilmente variabili da luogo a luogo, tanto da indurre la citata Lehmamm a ritenere la base del mantello alquanto accidentata.

Ammettendo l'esistenza del mezzo stratificato di transizione tra mantello e nucleo (di cui si è detto al punto II) queste irregolarita trovano piena giustificazione. Esse infatti possono provenire dalla variabilità del tragitto delle onde stesse nelle stratificazioni del mezzo di transizione sopra detto. 
(iò spiega lo sparpagriamento delle onde longitudinali e trasversali alla base del mantello, determinato appunto dalla stratificazione (forse non omogeneamente distribuita) del cuscinetto di transizione. Tale sparpagliamento si riflette naturalmente nei tempi di arrivo delle onde $P$ nella zona d'ombra (dromocrone come medie di tempi sensibilmente diversi), come da Fig. 5.

y) Honda e Hasaya sostengono che, nel caso più verosimile in cui il nucleo è considerato liquido, l'energia osservata per le onde ScS, corrisponde in media al $50 \%$ di quella calcolata (vedi $2 a$ ). Ciò dà una ulteriore conferma dell'esistenza dello strato di transizione tra mantello e nucleo. it da escludere infatti, come si ritiene dai suddetti autori, che il mezzo assorba il 50\% dell'energia dell'onda $S$ riflessa dal nucleo, in quanto è risaputo che esso influisce in misura molto minore.

Infatti è stato provato che il cocfficiente di attenuazione decresce rapidamente con la profondità $\left({ }^{8}\right)$ e che, in ogni caso, l'estinzione ammonta approssimativamente soltantio al 10-15 per cento dei valori che essa assume nella crosta terrestre $\left({ }^{9}\right)$.

Del resto, gli stessi autori sono costretti ad ammetterlo nella $3^{a}$ delle pubblicazioni citate (vedi $2 c)$ ).

$\grave{E}$ da supporre invece, che l'energia dell'onda $S$ incidente venga restituita per metà sotto forma di ScS (cioè riflessa dalla base del mantello) e per il restante $50 \%$ restituita in impulsi successivi sotto forma di $S_{N} S$, ossia riflessa dalle discontinuità della zona di transizione menzionata, associata a periodi più piccoli.

3. - Quanto è stato trovato da Caloi nel 1964 sulla base dell'osservazione delle onde $P c P, S c P, P c S$, ed onde associate (nuova notevole documentazione delle quali è stata qui riportata), ha ottenuto recente conferma per altra via $\left({ }^{10}\right)$.

Nel lavoro citato, avevo ritenuto come più probabile origine dello strato di transizione, quella contenuta nella teoria di Ramsey, concernente il passaggio di un mezzo elastico da una fase molecolare ad una fase metallica, per raggiunto limite della pressione critica.

Poiché, fino a qualche anno fa, la discontinuità di Wiechert era considerata brusca - un salto improvviso nel campo elastico e in quello della densità - per sostenere la sua ipotesi relativa al mutamento di fase per pressione (senza cambiamenti di natura chimica), Ramsey era stato costretto a postulare per il basso mantello l'esistenza di un mezzo chimicamente omogeneo ("The core discontinuity may ... be tentati- 
vely attributed to the excitation of the molecule $\left.\mathrm{SiO}_{2}\right)$ - pag. 421 di "On the Nature of the Earth's core») (11). Qualsiasi altra ipotesi, porta all'esistenza di uno strato di transizione, sia nel caso di mutamenti nella composizione chimica (pag. 415 l.c.), sia se si ritiene la graviti come fattore determinante: in questo caso, infatti, secondo Ramsey, non si avrebbe un salto brusco nella densiti ("jump in density"), ma un graduale aumento per lo spessore di $300 \mathrm{~km}$ almeno (pag. 415 l.c.). Sempre nell'ipotesi dell'esistenza di una superfieie di discontinuità alla base del mantello, Ramsey era stato condotto ad escludere la possibilitia, suggerita da qualche geochimico, sull'esistenza di uno strato di solfuri metallici alla base del mantello. In contrasto con i dati sismici - dice Ramsey a pag. 423 l.c. - " there should be two reflections, one at the top of the layer and one at the bottom. It is also impossible to explain the anomaly (just outside the core) as a mixing of the materials of the core and the mantle; this would be in contradiction with the observed sharpness of the boundary".

Del resto, la presumta esistenza di una superficie di discontinuita fra mantello e nucleo, ha costituito un valido argomento anche per gli avversari della teoria di Ramsey. Una volta provato - come sostengono Birch e numerosi geochimici - che il mantello è costituito da un sistema a più componenti (probabilmente, secondo Birch, ossidi compatti di magnesio, silicio e ferro), appariva insostenibile un mutamento di fase per alta pressione sotto forma di brusea discontinuiti. "A phase change in a multicomponent system almost certainly will be spread out over a range of pressures, whereas the core-mantle boundary presents "very sharp discontinuity" $\left({ }^{12}\right)$.

Il sospetto che la discontinuità fra mantello e nucleo non fosse una superfieie matematica era gia stato espresso da Sezawa e Kanai nel 1935, al termine di un notevole lavoro sulla trasmissione e riflessione delle onde elastiche $\left({ }^{13}\right)$. Essi osservano che "the discontinuity that forms the boundary of the earth's core, instead of having a geometrical sharp surface as imagined, is more than likely to be such that the intermediate layer will have a certain effective thickness, ... ".

Più recentemente, Bullard e Griggs $\left({ }^{14}\right)$, in uno studio sulla discontinuiti di Mohorovicic, concludono "that the transition should not be sharp in a rock which is not composed of a single well defined ehemical substance".

In ogni modo, sulla base di quanto ho esposto nel citato lavoro del 1964 , e delle ulteriori testimonianze qui riportate, non solo la zona di transizione fra mantello e nucleo esiste, ma probabilmente è, a sual volta, 
stratificata, come sembrano provarlo i numerosi echi che da essa prorengono (Vedi Figg. 1-4).

Come si sono formati? Mi sembra che la teoria di Ramsey sia ancora la più idonea a spiegarlo.

Si sa che, quando un gas è portato allo stato solido, le sue molecole si distribuiscono ordinatamente in un reticolo cristallino. La loro possibilità di movimento viene, in conseguenza, ridotta ad un volume molto ristretto intorno alla posizione relativa al reticolo stesso. A pressioni maggiori, i legami molecolari si rompono e il reticolo viene occupato da atomi, anziché da molecole. Lo stato solido alle alte pressioni può quindi passare dalla fase molecolare alla fase atomica, detta anche metallica.

Fon mi soffermerò naturalmente su questioni inerenti alla struttura della materia, anche perchè non rientra nelle mie competenze. ì noto che un metallo di volume $V$ può essere considerato come una scatola di ugual volume contenente $N$ elettroni. Per il principio di esclusione di Pauli, soltanto due di questi elettroni possono sussistere in ogni stato previsto dall'equazione dei livelli energetici della scatola in questione. Tutti questi elettroni tendono a raceogliersi, nel più basso stato di energia, formando il cosi detto gas di Fermi (*). Gli $N$ elettroni occuperanno tutti gli stati energetici, dallo stato più basso allo stato di energia cinetica $K E_{o}$, detto livello di Fermi:

$$
K E_{o}=\frac{h^{2}}{8 m}\left(\frac{3}{\pi} N\right)^{2 / 3},
$$

dove $h$ è la costante di Plank, $m$ la massa ed $N$ il numero di elettroni liberi per centimetro cubico.

Lo studio della relazione pressione-densità della materia nelle cennate eondizioni è ricondotto alla ricerca della relazione pressione-volume molecolare o atomico, la densità essendo strettamente legata a quest'ultimo. Dopo la fondamentale ricerea di Krönig, De Boer e Korringa (1946), su questa strada si è messo, come è noto, Ramsey, il quale è giunto alla conclusione che l'olivina, alla base del mantello, si trova nelle condizioni di pressione atte a provocarne il passaggio dalla fase molecolate alla fase atomica (metallica).

In un solido composto da joni con livelli saturati (reticolo cristallino), al crescere della pressione si raggiunge un valore critico per il

(*) Vedi p. es. (15). 
quale il lavoro delle forze che tendono a comprimere il solido nello stato più denso, uguaglia quello richiesto dalla rottura dei legami elettronici.

Naturalmente, dopo il primo passaggio alla nuova densità, non è detto che il solido sia stabile per qualsiasi pressione maggiore di quella eritica. Se con un determinato valore della pressione si sono spezzati $i$ legami fra elettroni dei livelli occupati più estemi, aument ando ulteriormente, la pressione $P$ raggiungera un nuovo valore critico, con conseguente ripetizione del processo accennato, sicehé la relazione pressionedensità avrà un andamento analogo a quello schematizzato nella Fig. 7.

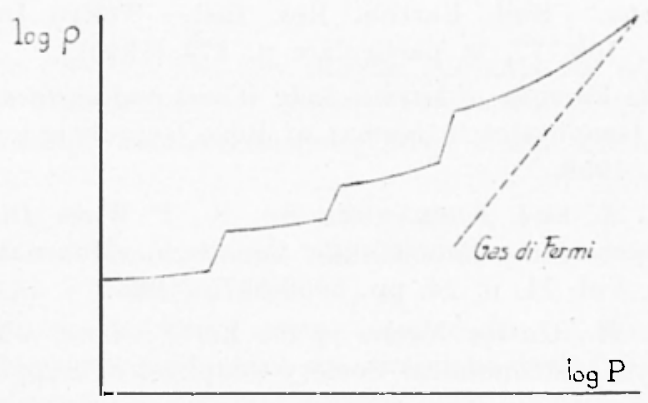

Fig. 7

Quando la pressione raggiunge un valore talmente elevato, capace di privare di elettroni tutti i livelli elettronici, abbiamo la formazione del gas di Fermi.

In un sistema a più componenti, il processo sommariamente accennato, spiega la formazione della zona di transizione fra mantello e nucleo, nonché la sua probabile stratificazione.

\section{BIBLIOGRAFIA}

(1) Calor P., Sulle reali dimensioni del nucleo terrestre, "Rend. Acc. Naz. dei Lincei "(Classe Sc. fis. mat. o nat.), ser. VIII, Vol. XXXVI, fasc. 7 (1964).

(2) Calor P., On the Upper Mantle, in "Advances in Geophysics", Vol. 12, Academic Press, New York (in preparazione).

${ }^{3}$ ) Calor P., Sulla velocilà di propagazione delle onde longiludinali alla base del mantello terrestre, "Rend. Acc. Naz. dei Lincei" (Classe Sc. fis. mat. e nat.), ser. VIII, Vol. XXXII, fase. 2 (1962).

(4) HoNDA H., On the ScS waves and the rigidity of the Earth's core. "The Central Meteorological Observatory" pp. 165-177 (1934). 
(5) HONDA H. and HASAYA Y., On the SeS waves and the rigidity of the Earth's core (Second paper). "The Central Meteorological Observatory" pp. 219 $230(1940)$.

(6) HONDA H. and Ito II., On the reflected waves from deep focus earthquakes. "The Science Reports of the Tohoku University", Series 5, Geophysics, Vol. 3, 11. 3, pp. 144-155 (1951).

(7) HoNda H., Sma II. and Nakayura K., The SeS waves, the mechanism of deep earthqualie and rigidity of the earth's core. "The Science Reports of the Tohoku Eniversity". Series 5, Geophysics, Vol. 7, 1. 3, pp. 170179 (1956).

(9) Sezawa K. and Kaxal K., Tiscosity distribution within the Earth. Preliminary Noles. "Bull. Earthq. Res. Inst." Tokyo Inp. Univ., Vol. XVIII, pp. 169-177, in particolare p. 173 (1940).

(") BATI M., The Energies of seismic body Waves and surface Waves "Contributions in Geophysics in honour of Beno Gutenberg ". pp. 1-16. Pergamon Press, 1958.

(10) Puixiey R. A. and Alexaxuer Sh. S., P Wave Diffraction Theory and Structure of the Core-Manlle Boundary, "Journal of Geophysical Research ", Vol. 71, 1. 24, pp. 5959-5975 (1966).

(11) Raysey W. II., On the Nature of the E'arth's Core, "Monthly Notices of the Royal Astronomical Society-Geophysical Supplement", Vol. 5, n. 9 (1949).

(12) J A Cons J. A., The Earth's Core and Geomagnetism. Pergamon Press, Jondon (1963), p. 25.

(13) SEzawa K. and KaxaI K., The Effect of Sharpness of Discontinuilies on the Transmission and Reflection of Elastic waves. "Bull. Earthq. Research Institute ", Tokyo Imp. University, Vol. XIII, n. 4, p. 755. (1935).

(is) Bulinird E. C. and Griges D. T., The Nature of the Mohorovicic Discontinuily, "Geophyss. Journ. of the Royal Astr. Soc. ", Vol. 6, n. 1, pp. 118. 123 (1961).

(15) Orear J., Fundamental Physies, Cap. 13 "Atomic Theory ". John Wiley, New York (1963). 